\title{
Selective white matter abnormalities in a novel rat model of vascular dementia.
}

\section{$\operatorname{AUTHOR}(\mathrm{S}):$}

Kitamura, Akihiro; Fujita, Youshi; Oishi, Naoya; Kalaria, Raj $\mathrm{N}$; Washida, Kazuo; Maki, Takakuni; Okamoto, Yoko; ...

Fukuyama, Hidenao; Takahashi, Ryosuke; Ihara, Masafumi

\section{CITATION:}

Kitamura, Akihiro ...[et al]. Selective white matter abnormalities in a novel rat model of vascular dementia.. Neurobiology of aging 2012, 33(5): 1012.e25-1012.e35

\section{ISSUE DATE:}

2012-05

URL:

http://hdl.handle.net/2433/154844

\section{RIGHT:}

C 2012 Elsevier Inc:; この論文は出版社版でありません。引用の際には 出版社版をご確認ご利用ください。; This is not the published version. Please cite only the published version. 


\section{Selective white matter abnormalities in a novel rat model of vascular dementia}

Akihiro Kitamura $^{\mathrm{a}}$, Youshi Fujita ${ }^{\mathrm{a}}$, Naoya Oishi ${ }^{\mathrm{b}}$, Raj N Kalaria ${ }^{\mathrm{c}}$, Kazuo Washida ${ }^{\mathrm{a}}$, Takakuni

Maki $^{\mathrm{a}}$, Yoko Okamoto ${ }^{\mathrm{a}}$, Yoshiki Hase ${ }^{\mathrm{a}}$, Mahito Yamada ${ }^{\mathrm{a}}$, Jun Takahashi ${ }^{\mathrm{d}}$, Hidefumi Ito ${ }^{\mathrm{a}}$, Hidekazu Tomimoto $^{\mathrm{e}}$, Hidenao Fukuyama ${ }^{\mathrm{b}}$, Ryosuke Takahashi $^{\mathrm{a}}$, Masafumi Ihara ${ }^{\mathrm{a}}$

${ }^{a}$ Department of Neurology, Graduate School of Medicine, Kyoto University, Sakyo-ku, Kyoto, Japan, ${ }^{\text {H} H u m a n ~ B r a i n ~ R e s e a r c h ~ C e n t e r, ~ G r a d u a t e ~ S c h o o l ~ o f ~ M e d i c i n e, ~ K y o t o ~ U n i v e r s i t y, ~ K y o t o, ~}$ Japan, ${ }^{C}$ Institute for Ageing and Health, WRC, Campus for Ageing and Vitality, Newcastle University, UK, ${ }^{\mathrm{d}}$ Department of Biological Repair, Institute for Frontier Medical Sciences, Kyoto University, Kyoto, Japan, ${ }^{\mathrm{e}}$ Department of Neurology, Graduate School of Medicine, Mie University, Tsu, Japan

Correspondence to Masafumi Ihara, MD, Kyoto University, 54 Kawahara-cho, Shogoin, Sakyo, Kyoto 606-8507, Japan. Telephone:(+81)-75-751-3766, Fax:(+81)-75-751-3265,

E-mail:ihara@kuhp.kyoto-u.ac.jp 


\section{Abstract}

Rats subjected to bilateral common carotid artery (CCA) occlusion or 2-vessel occlusion $(2 \mathrm{VO})$ have been used as animal models of subcortical ischemic vascular dementia. However, this model possesses an inherent limitation in that cerebral blood flow (CBF) drops too sharply and substantially after ligation of CCAs. To circumvent such hypoxic-ischemic conditions, we tested to implant the ameroid constrictor device on bilateral CCAs of male Wistar-Kyoto rats and more precisely replicated chronic cerebral hypoperfusion by gradual narrowing of the CCAs (2-vessel gradual occlusion; 2VGO). The acute CBF reduction and resultant inflammatory responses observed in the $2 \mathrm{VO}$ rats were eliminated in the $2 \mathrm{VGO}$ rats. Thus, chronic cerebral hypoperfusion was segregated, and induced selective white matter changes with relatively preserved neurovascular coupling and substantially less metabolic and histological derangements in the gray matter including the hippocampus. This led to significant spatial working memory impairment of a similar magnitude to the $2 \mathrm{VO}$ rats at 28 days post-operation. The 2VGO model may more closely mimic cognitive impairment subsequent to selective white matter damage.

Keywords: Vascular dementia; Chronic cerebral hypoperfusion; Ameroid constrictor; White matter change; Rat; Neurovascular coupling 


\section{Introduction}

Vascular dementia $(\mathrm{VaD})$ is the second most common cause of dementing illnesses after

Alzheimer's disease (AD). Approximately half of all cases of VaD are explained by subcortical ischemic vascular dementia (SIVD) (Yoshitake et al., 1995), which is characterized by lacunar infarctions in the basal ganglia and ischemic white matter changes. Loss of vasomotor reactivity in the small vessels and resultant chronic cerebral hypoperfusion with blood-brain barrier (BBB) disruption and glial activation may underlie the white matter changes (Marshall and Lazar, 2011; Pantoni, 2010).

To mimic such a pathological condition and explore the underlying mechanisms, several animal models of chronic cerebral hypoperfusion have been developed, including bilateral common carotid artery (CCA) or 2-vessel occlusion (2VO) in rats, bilateral CCA stenosis in gerbils or mice (BCAS) (Shibata et al., 2004) and unilateral CCA occlusion in mice (Yoshizaki et al., 2008). Among these, the rat $2 \mathrm{VO}$ model has been frequently used (Jiwa et al., 2010) and may become more important once genetically-modified rats are widely available. The $2 \mathrm{VO}$ model exhibits characteristic features of SIVD, such as white matter damage (Wakita et al., 1994; Wakita et al., 2002), and cognitive impairment (Farkas et al., 2007; Hainsworth and Markus, 2008; Jiwa et al., 2010). Nevertheless, this model possesses an inherent limitation in that cerebral blood flow (CBF) drops too sharply and substantially after the ligation of the 
CCAs and remains too low (30-45\% of the baseline level) for 2-3 days; this therefore creates hypoxic-ischemic conditions too severe to replicate 'chronic' cerebral hypoperfusion (Marosi et al., 2006). Following this acute phase, $\mathrm{CBF}$ gradually recovers but still remains relatively low (50-90\%) for 8 weeks to 3 months post-operation (chronic phase) (Otori et al., 2003; Tomimoto et al., 2003). Although the sustained oligemia in the chronic phase is believed to better replicate chronic cerebral hypoperfusion associated with SIVD, the contribution of the preceding acute phase to the neuropathological and behavioral consequences is an ongoing concern (Farkas et al., 2007). We therefore sought to establish a novel rat model that eliminates the acute phase, meaning that $\mathrm{CBF}$ would gradually decrease to the level in the chronic phase, using the ameroid constrictor device. This device could predictably achieve gradual narrowing of the CCAs and establish a rat model of SIVD that replicates 'chronic' cerebral hypoperfusion more precisely. We therefore henceforth refer to this novel rat model as the '2-vessel gradual occlusion (2VGO)' model.

\section{Materials and Methods}

\section{1. Animals}

We used 12- to 14-week-old male Wistar-Kyoto rats (WKY) (See Supplemental Method I for details). 


\section{2. Ameroid constrictor}

The ameroid constrictor (Research Instruments NW, OR, USA) consists of a stainless steel casing surrounding a hygroscopic casein material that has an internal lumen. The casein component gradually absorbs water and consequently swells, leading to predictable narrowing and occlusion of the arterial lumen that it encases (Fig. 1A, B and C). The inner diameter was $0.5 \mathrm{~mm}$, the outer diameter $3.25 \mathrm{~mm}$ and the length $1.28 \mathrm{~mm}$.

\section{3. Study design}

WKY rats were divided into three groups: 2-vessel gradual occlusion (2VGO), 2-vessel occlusion (2VO) and sham control groups (Supplemental Method II). Temporal changes of blood flow in the CCAs were recorded by computed tomography-angiography (CTA); the CBF in the frontal cortices was measured by laser speckle flowmetry (LSF; Omega Zone;

Omegawave, Tokyo, Japan), and ${ }^{18} \mathrm{~F}$-Fluorodeoxyglucose (FDG) positron emission tomography (PET) imaging was performed at indicated time points. Histological evaluation of demyelinating changes and glial activation, analysis of cerebral mRNA and protein levels of inflammatory cytokines and Western blotting analysis of myelin basic protein (MBP) were performed at indicated points. Spatial working memory was assessed by the Y-maze test (Fig. 2).

\section{4. Surgical procedure}


Under anesthesia, the 2VGO rats were subjected to surgical implantation of ameroid constrictors on the CCAs bilaterally whereas in the 2VO group, both the CCAs were ligated (Supplemental Method III).

\section{5. Computed tomography-angiography}

CTA of the bilateral CCAs was repeatedly performed under anesthesia using the $\mathrm{R} \_\mathrm{mCT}$ system (Rigaku, Tokyo, Japan) (Supplemental Method IV).

\section{6. Cerebral blood flow, blood pressure and pulse rate measurement}

CBF in the frontal cortices was recorded by LSF under anesthesia with isoflurane (1.5\%) (Supplemental Method V).

\section{7. ${ }^{18}$ F-FDG PET acquisition and image analysis}

Small animal PET imaging was performed to assess temporal changes in ${ }^{18} \mathrm{~F}$-FDG uptake during the first 5 minutes and again between 45 and 90 minutes (Supplemental Method VI).

\section{8. Histological evaluation of demyelinating changes and glial activation}

Paraffin-embedded coronal sections of the brain ( $6 \mu \mathrm{m}$-thick) were analyzed with hematoxylin-eosin (HE) staining for detection of infarcted areas in the forebrain and for neurodegeneration in the hippocampal CA1 and CA3 regions, and with Klüver-Barrera staining for demyelinating changes. The severity of the white matter lesions in the optic chiasma, medial and lateral parts of the corpus callosum and caudoputamen, was graded as normal (Grade 0), 
disarrangement of the nerve fibers (Grade 1), the formation of marked vacuoles (Grade 2) and the disappearance of myelinated fibers (Grade 3), according to the established criteria (Wakita et al., 1994). They were also immunostained for ionized calcium binding adaptor molecule-1 (Iba-1; a marker of microglia; Wako, Osaka, Japan), glial fibrillary acidic protein (GFAP; astrocyte; DAKO, Glostrup, Denmark), glutathione S-transferase- $\pi$ (GST- $\pi$; oligodendrocyte; Assay Designs, Ann Arbor, MI, USA), degraded MBP (dMBP; demyelinating changes; a gift from Dr. Wakita) (Ihara et al., 2010) and myelin-associated glycoprotein (MAG; Santa Cruz Biotechnology, CA, USA) (Supplemental Method VII).

\section{9. Quantitative real-time reverse transcriptase-polymerase chain reaction}

Cerebral mRNA levels of the monocyte chemoattractant protein-1 (MCP-1) and tumor necrosis factor- $\alpha$ (TNF- $\alpha$ ) were assessed by quantitative real-time RT-PCR (Supplemental Method VIII).

\section{10 Enzyme-Linked Immunosorbent Assay (ELISA) for TNF- $\alpha$}

The protein concentration of TNF- $\alpha$ in brain homogenates were also measured using a specific ELISA kit according to the manufacturer's recommendation (R\&D systems,

Minneapolis, MN, USA) (Supplemental Method IX).

2. 11. Western blotting analysis for myelin basic protein

The optic nerves and chiasma were analyzed for demyelinating changes. A rabbit anti-MBP 
antibody and a mouse anti $\beta$-actin antibody (which served as an internal control), were used

(Supplemental Method X).

2. 12. Y-maze test for spatial working memory assessment

Spatial working memory was assessed by the Y-maze test (Supplemental Method XI).

\section{13. Statistical analysis}

All values are expressed as mean \pm SEM. One-way analysis of variance was used to evaluate significant differences among groups (except where otherwise stated) followed by a post hoc Tukey test or Tukey-Kramer test. Differences with $P<0.05$ were considered significant in all statistical analyses.

\section{Results}

\section{1. Mortality rates and body weight changes of rats}

The mortality rate in the 2 VGO group was $2.0 \%(1 / 49)$. This was much lower than that of the $2 \mathrm{VO}$ group, which was $13.8 \%$ (8/58), in accordance with previous reports (Fujishima et al., 1976). In the sham group, all of 48 rats survived until euthanized. Body weight decreased in both the $2 \mathrm{VGO}$ and $2 \mathrm{VO}$ groups, which was significantly less severe in the $2 \mathrm{VGO}$ group at 1 day post-operation (2VGO group, $-14.1 \pm 2.0 \%$ vs. 2 VO group, $-27.4 \pm 1.7 \% ; P<0.01$ ). Both groups of rats started to regain body weight at 3 days. 


\section{2. Gradual narrowing of the common carotid arteries}

Bilateral placement of the ameroid constrictors on the CCAs produced the expected narrowing of the inner lumen: $0.28 \mathrm{~mm}$ at 1 day post-operation, $0.24 \mathrm{~mm}$ at 3 days and $0.19 \mathrm{~mm}$ at 5 days (Fig. 1B and C). CTA indicated that blood flow of CCAs distal to the implanted ameroid constrictors did not decrease at 1 hour but started to decrease at 3 hours, leading to complete occlusion of the CCAs at 3 days (Fig. 3).

\section{3. Temporal profiles of $C B F$ recorded by $L S F$}

In the $2 \mathrm{VO}$ group, $\mathrm{CBF}$ dropped sharply to $55.2 \pm 0.8 \%$ of the baseline level at 3 hours post-operation but started to recover at 3 days and reached to $85.6 \pm 3.7 \%$ at 28 days. In contrast, in the 2VGO group, the acute phase observed in the 2VO group was absent; CBF did not decrease at 1 hour $(102.5 \pm 2.9 \%)$ but started to decrease at 3 hours $(79.6 \pm 3.3 \%)$, and reached a minimum at 3 days $(69.3 \pm 1.7 \%)$, before gradually recovering at 28 days $(84.5 \pm 3.3 \%)$. In the sham group, no apparent change of CBF was detected. Two-way repeated-measures analysis of variance showed a significant interaction between group and time $(\mathrm{F}(14,63)=16.976, P<0.001)$ and significant differences between the 2VGO and 2VO groups at 1, 3 and 6 hours and 1 day (Fig. 4A and B).

The blood pressure and pulse rate of the surviving rats did not differ significantly among the three groups at any postoperative intervals until 28 days. 


\section{3. $4 .{ }^{18}$ F-FDG PET analysis}

The first 5-minute uptake of ${ }^{18} \mathrm{~F}-\mathrm{FDG}$, which was used as an indicator of $\mathrm{CBF}$, showed a similar temporal profile of the CBF recorded by LSF: the uptake dropped more substantially in the $2 \mathrm{VO}$ than $2 \mathrm{VGO}$ groups at 3 hours post-operation but recovered at 28 days in the both groups (Fig. 5A).

The late ${ }^{18} \mathrm{~F}$-FDG uptake, used as an indicator of cerebral glucose metabolism, did not significantly decrease at 28 days in the $2 \mathrm{VGO}$ group. Whereas, in the $2 \mathrm{VO}$ group at 28 days, late ${ }^{18}$ F-FDG uptake significantly decreased in the cerebral cortex compared to that in the 2VGO group and in the hippocampus compared to pre-operation (Fig. 5B).

\section{5. Y-maze test for spatial working memory assessment}

In the $2 \mathrm{VGO}$ and $2 \mathrm{VO}$ groups, the percentage of alternation behaviors (used as a reflection of spatial working memory) was significantly decreased (Fig. 6A), whereas the number of arm entries was significantly increased, compared with the sham group at 28 days (Fig. 6B).

\section{6. Histological analysis of gray matter damage}

Brain infarcted areas were not found in any rats of the $2 \mathrm{VGO}$ and sham groups but in $50 \%$ of rats in the 2VO group at 3,14 and 28 days (Supplemental Fig. 1A). The infarctions were distributed mainly in the caudoputamen. Cortical gliosis was not found in the sham group and was present in only $8.3 \%$ of rats in the 2 VGO group at 3,14 and 28 days (Supplemental Fig. 
1B); however, cortical gliosis was present in $75 \%$ of rats in the $2 \mathrm{VO}$ group over the same

periods. There was a trend toward increased density of pyknotic neurons in the hippocampal

CA1 region at 28 days in the $2 \mathrm{VO}$ group compared to the $2 \mathrm{VGO}$ and sham groups

(Supplemental Fig. 1C); however, there were no significant differences in density among the three groups.

\section{7. Histological analysis of white matter damage}

Quantitative analysis of the grading score of Klüver-Barrera staining of the corpus

callosum indicated that the white matter damage was significantly more severe in both the $2 \mathrm{VGO}$ and $2 \mathrm{VO}$ groups compared with the sham group at 28 days post-operation; however, the extent of damage was significantly smaller in the $2 \mathrm{VGO}$ compared to $2 \mathrm{VO}$ groups (Fig. 7A).

Similar findings in the degree of myelin damage (assessed by dMBP staining) (Fig. 7B) and the density of GST- $\pi$-positive oligodendrocytes (Fig. 7C) were discovered: the white matter damage was slightly milder in the $2 \mathrm{VGO}$ compared to the $2 \mathrm{VO}$ groups but was still more severe than the sham group. In the $2 \mathrm{VGO}$ and $2 \mathrm{VO}$ groups, MAG-immunopositive regions (indicating the degree of axon-glial integrity) decreased compared to the sham group, with the most apparent difference at 28 days (data not shown). The density of GFAP-immunopositive astrocytes was significantly greater in both the $2 \mathrm{VGO}$ and $2 \mathrm{VO}$ groups compared to the sham group at 28 days (data not shown). The density of Iba-1-immunopositive microglia increased from 1 day in the 
$2 \mathrm{VO}$ group; while in the $2 \mathrm{VGO}$ group, there was no change in microglia density at 1 day but an increase at 3 days. The microglial density in both the 2VGO and $2 \mathrm{VO}$ groups remained significantly greater than the sham group at 28 days (Supplemental Fig. 2A and B).

\section{8. Western blotting analysis for MBP at 28 days post-operation}

In addition to dMBP immunohistochemistry, MBP immunoblotting was evaluated as a marker of white matter integrity. Western blotting for MBP at 28 days showed multiple bands corresponding to the four isoforms $(21.5 / 18.5 / 17 / 14 \mathrm{kDa})$. Densitometric analyses showed significant reduction in those bands both in the $2 \mathrm{VGO}$ and $2 \mathrm{VO}$ groups compared to the sham group. However, the reduction was less substantial in the 2VGO than 2VO groups (Fig. 8).

\section{9. $m R N A$ expression of inflammatory cytokines at 1 day post-operation}

At 1 day, cerebral mRNA expression of inflammatory cytokines, MCP- 1 and TNF- $\alpha$, was significantly increased in the $2 \mathrm{VO}$ group but slightly increased in the 2VGO group compared to the sham group (Fig. 9A and B).

3. 10. The protein level of TNF- $\alpha$ at 1 day post-operation.

At 1 day, cerebral protein level of TNF- $\alpha$ was significantly increased in both the $2 \mathrm{VGO}$ and $2 \mathrm{VO}$ groups compared to the sham group, with a tendency of increase in the $2 \mathrm{VO}$ group (Fig. 9C). 


\section{Discussion}

The findings in this study suggest circumvention of the acute phase of CBF reduction and resultant acute inflammatory response observed in the $2 \mathrm{VO}$ model by gradual narrowing of the bilateral CCAs using the ameroid constrictor device instead of ligation. As a result, segregation of the chronic phase from the acute phase was achieved, suggesting a more precise reconstruction of chronic cerebral hypoperfusion evident in clinical cases of SIVD. This method induced demyelinating changes with inflammatory and glial responses but without apparent infarcted lesions or pronounced metabolic derangement in the gray matter. White matter changes were substantial in both the $2 \mathrm{VGO}$ and $2 \mathrm{VO}$ groups but significantly less severe in the 2VGO group. This suggests that the neuropathological consequences and metabolic changes of the $2 \mathrm{VO}$ model had been generated not only by chronic cerebral hypoperfusion but also by acute drop in CBF immediately after bilateral CCAs occlusion. The differences in mortality rate, infarction rate and body weight loss between the $2 \mathrm{VGO}$ and $2 \mathrm{VO}$ groups may reflect gradual $\mathrm{CBF}$ reduction in the $2 \mathrm{VGO}$ group.

Y-maze tests showed significant spatial working memory impairment in both the $2 \mathrm{VGO}$ and $2 \mathrm{VO}$ groups. The spatial working memory impairment in the 2VGO model may be derived from white matter damage because neuropathological changes and glucose hypometabolism were not apparent in the cortex and hippocampus at 28 days. By contrast, the $2 \mathrm{VO}$ model 
exhibited persistent metabolic derangement in the gray matter at 28 days, despite CBF recovery; this suggests neurovascular uncoupling even at 28 days post-operation. The $2 \mathrm{VO}$ and $2 \mathrm{VGO}$ model may thus be defined by the presence or absence of the acute CBF drop, respectively; therefore, the persistent neurovascular uncoupling at 28 days in the $2 \mathrm{VO}$ group may result from the ischemic insult applied to the brain in the acute phase post-operation. These results therefore suggest that the $2 \mathrm{VGO}$ model may more closely replicate the condition of working memory impairment subsequent to ischemic white matter changes.

The reasons as to why the $2 \mathrm{VGO}$ model showed comparable spatial working memory impairment to the $2 \mathrm{VO}$ model may be explained by the fact that less pronounced white matter damage was sustained in the $2 \mathrm{VGO}$ model compared to the $2 \mathrm{VO}$ model where the damage was sufficient to induce spatial working memory impairment detected by the Y-maze test. Another reason may be the impaired glial-axon integrity (assessed with MAG immunostaining) (Quarles, 2007), which was comparable between 2 VGO and 2VO models; this therefore may have contributed to the working memory impairment.

No single animal model appears to recapitulate all the features of SIVD as humans have different brain anatomy and longer perforating arteries. In addition, the current model lacks vascular risk factors and causative small vessel changes that may lead to alterations in blood flow and cerebral autoregulation. One of the ways to resolve this problem could be to implant 
ameroid constrictors in spontaneous hypertensive rats (SHR). However, SHRs subjected to the bilateral CCA occlusion have shown more extensive CBF reduction accompanied by more extensive cerebral infarcts and demyelinating changes and higher mortality rate (72\%; 78 of 108) than the corresponding normotensive counterpart WKY rats $(16 \% ; 7$ of 43 ) (Fujishima et al., 1976; Ogata et al., 1976). Beside the high mortality, such severe CBF reduction with marked infarctions may not adequately represent SIVD. Our preliminary data showed that the mortality rate of SHRs subjected to implantation of ameroid constrictors to the bilateral CCAs was only $6.2 \%$ ( 1 of 16) (Kitamura, unpublished data). Therefore, comparison of the effects of 2VGO on SHRs and on WKY rats could facilitate our understanding of the clinical and pathological substrates of SIVD with small vessel pathology, with the caveat that the genetic background of SHR is not necessarily identical to that of WKY rats (St Lezin et al., 1992).

Thus far, rats with several different backgrounds have been used for 2VO model, including Sprague-Dawley, Wistar and WKY rats. There may therefore have been discrepancies between rats in terms of the temporal CBF profile after 2VO. The relative CBF reduction from the baseline level in the current study was smaller than that found in Sprague-Dawley or Wistar rats but approximated that of WKY rats (Farkas et al., 2007).

The ameroid constrictor has been applied to the porcine or canine coronary arteries to simulate atherosclerotic coronary heart disease (Litvak et al., 1957), and the rabbit and rat 
femoral arteries to mimic chronic hindlimb ischemia (Tang et al., 2005). However, this is the first report that the ameroid constrictor is applied to the CCA to replicate chronic cerebral hypoperfusion. Although the ameroid constrictor device achieves predictable gradual narrowing of the artery, the speed of arterial narrowing is dependent on both the size of ameroid constrictor and the diameter of arteries. As such, an in-vivo non-invasive imaging technique, such as CTA, may be applicable in the evaluation of the temporal change in carotid blood flow. Moreover, use of titanium for casing, instead of the stainless steel, will enable magnetic resonance imaging/angiography.

Rats of 12-14 weeks of age were used in the current study to reproduce chronic cerebral hypoperfusion. However, application of the ameroid constrictor to older animals for longer period should be considered given that SIVD is an age-related disease that develops over many years.

In conclusion, we have established a novel rat model that may more closely mimic cognitive impairment subsequent to selective white matter damage. The 2VGO model may therefore be adapted to explore possible treatments for SIVD.

\section{Disclosure statement}

The authors declare no conflict of interest. 


\section{Acknowledgments}

We thank Dr. Matsuo and Dr. Wakita for generously giving us the anti-dMBP antibody, and Dr. Khundakar for insightful comments and editing. We are indebted to Ms. Tanigaki, Ms.

Hikawa, Ms. Nakabayashi, Ms. Katsukawa and Mr. Kubota for their excellent technical assistance. This work was supported by grants from the Suzuken Memorial Foundation (to M.I. and J.T.) and the Japanese Vascular Disease Research Foundation (M.I.). R.N.K.'s research work is supported by the Medical Research Council, UK. 


\section{References}

Farkas E., Luiten P.G., Bari F., 2007. Permanent, bilateral common carotid artery occlusion in the rat: a model for chronic cerebral hypoperfusion-related neurodegenerative diseases. Brain Res Rev 54, 162-80.

Fujishima M., Ogata J., Sugi T., Omae T., 1976. Mortality and cerebral metabolism after bilateral carotid artery ligation in normotensive and spontaneously hypertensive rats. J Neurol Neurosurg Psychiatry 39, 212-7.

Hainsworth A.H., Markus H.S., 2008. Do in vivo experimental models reflect human cerebral small vessel disease? A systematic review. J Cereb Blood Flow Metab 28, 1877-91.

Ihara M., Polvikoski T.M., Hall R., Slade J.Y., Perry R.H., Oakley A.E., Englund E., O'Brien J.T., Ince P.G., Kalaria R.N., 2010. Quantification of myelin loss in frontal lobe white matter in vascular dementia, Alzheimer's disease, and dementia with Lewy bodies. Acta Neuropathol 119, 579-89.

Jiwa N.S., Garrard P., Hainsworth A.H. Experimental models of vascular dementia and vascular cognitive impairment: a systematic review. J Neurochem, 2010/08/25 Edition, Vol. 115; 2010. p 814-28.

Lin J.X., Tomimoto H., Akiguchi I., Wakita H., Shibasaki H., Horie R., 2001. White matter lesions and alteration of vascular cell composition in the brain of spontaneously hypertensive rats. Neuroreport 12, 1835-9.

Litvak J., Siderides L.E., Vineberg A.M., 1957. The experimental production of coronary artery insufficiency and occlusion. Am Heart J 53, 505-18.

Marosi M., Rakos G., Robotka H., Nemeth H., Sas K., Kis Z., Farkas T., Lur G., Vecsei L., Toldi J., 2006. Hippocampal (CA1) activities in Wistar rats from different vendors. Fundamental differences in acute ischemia. J Neurosci Methods 156, 231-5.

Marshall R.S., Lazar R.M., 2011. Pumps, aqueducts, and drought management: vascular physiology in vascular cognitive impairment. Stroke 42, 221-6.

Ogata J., Fujishima M., Morotomi Y., Omae T., 1976. Cerebral infarction following bilateral carotid artery ligation in normotensive and spontaneously hypertensive rats: a pathological study. Stroke 7, 54-60.

Otori T., Katsumata T., Muramatsu H., Kashiwagi F., Katayama Y., Terashi A., 2003. Long-term measurement of cerebral blood flow and metabolism in a rat chronic hypoperfusion model. Clin Exp Pharmacol Physiol 30, 266-72. 
Pantoni L., 2010. Cerebral small vessel disease: from pathogenesis and clinical characteristics to therapeutic challenges. Lancet Neurol 9, 689-701.

Quarles R.H., 2007. Myelin-associated glycoprotein (MAG): past, present and beyond. J Neurochem 100, 1431-48.

Roman G.C., Erkinjuntti T., Wallin A., Pantoni L., Chui H.C., 2002. Subcortical ischaemic vascular dementia. Lancet Neurol 1, 426-36.

Shibata M., Ohtani R., Ihara M., Tomimoto H., 2004. White matter lesions and glial activation in a novel mouse model of chronic cerebral hypoperfusion. Stroke 35, 2598-603.

St Lezin E., Simonet L., Pravenec M., Kurtz T.W., 1992. Hypertensive strains and normotensive 'control' strains. How closely are they related? Hypertension 19, 419-24.

Tang G.L., Chang D.S., Sarkar R., Wang R., Messina L.M., 2005. The effect of gradual or acute arterial occlusion on skeletal muscle blood flow, arteriogenesis, and inflammation in rat hindlimb ischemia. J Vasc Surg 41, 312-20.

Tomimoto H., Ihara M., Wakita H., Ohtani R., Lin J.X., Akiguchi I., Kinoshita M., Shibasaki H., 2003. Chronic cerebral hypoperfusion induces white matter lesions and loss of oligodendroglia with DNA fragmentation in the rat. Acta Neuropathol 106, 527-34.

Wakita H., Tomimoto H., Akiguchi I., Kimura J., 1994. Glial activation and white matter changes in the rat brain induced by chronic cerebral hypoperfusion: an immunohistochemical study. Acta Neuropathol 87, 484-92.

Wakita H., Tomimoto H., Akiguchi I., Matsuo A., Lin J.X., Ihara M., McGeer P.L., 2002. Axonal damage and demyelination in the white matter after chronic cerebral hypoperfusion in the rat. Brain Res 924, 63-70.

Yoshitake T., Kiyohara Y., Kato I., Ohmura T., Iwamoto H., Nakayama K., Ohmori S., Nomiyama K., Kawano H., Ueda K., et al., 1995. Incidence and risk factors of vascular dementia and Alzheimer's disease in a defined elderly Japanese population: the Hisayama Study. Neurology 45, 1161-8.

Yoshizaki K., Adachi K., Kataoka S., Watanabe A., Tabira T., Takahashi K., Wakita H., 2008. Chronic cerebral hypoperfusion induced by right unilateral common carotid artery occlusion causes delayed white matter lesions and cognitive impairment in adult mice. Exp Neurol 210, 585-91. 


\section{Figure Legends}

\section{Figure 1.}

A, Surgical implantation of the ameroid constrictor device on the right common carotid artery.

B, Representative images of ameroid constrictors at indicated time points.

C, Table showing the temporal profiles of stenosis of ameroid constrictor before surgical

implantation $(n=4)$ and $1(n=8)$ and 3 days $(n=4)$ post-operation.

\section{Figure 2.}

Experimental protocol (d=day and $\mathrm{hr}=$ hour $)$.

\section{Figure 3.}

Three-dimensional volume-rendered images of CT-angiography showing the blood flow of the bilateral common carotid arteries pre- and post-implantation of ameroid constrictors at indicated time intervals. Arrowheads indicate common carotid arteries and arrows ameroid constrictors.

\section{Figure 4.}

A, Representative CBF images of laser speckle flowmetry in each group at indicated time intervals. The value in each image indicates relative CBF that is expressed as a percentage $( \pm$ SEM) of the baseline level (100\%). Four to six animals in each group were used at each point.

B, The temporal profile of $\mathrm{CBF}$ in each group. $* P<0.05$, ** $P<0.01,2 \mathrm{VGO}$ vs. $2 \mathrm{VO}$.

\section{Figure 5.}


A, Representative ${ }^{18}$ F-FDG PET coronal images superimposed on MR images for the first 5 minutes of the 2VGO (upper left panels) and 2VO (upper right panels) groups. Temporal profiles of the mean normalized ${ }^{18}$ F-FDG count during the first 5 minutes in the cerebral cortex (lower left), caudoputamen (lower middle) and hippocampus (lower right) at indicated time intervals.

B, Representative ${ }^{18}$ F-FDG PET coronal images superimposed on MR images between 45 and 90 minutes of the $2 \mathrm{VGO}$ (upper left panels) and 2VO (upper right panels) groups. Temporal profiles of the mean normalized ${ }^{18} \mathrm{~F}$-FDG count between 45 and 90 minutes in the cerebral cortex (lower left), caudoputamen (lower middle) and hippocampus (lower right) at indicated time intervals. Three to four animals in each group were used at each point. * $P<0.05$, ** $P<0.01,2$ VGO vs. 2 VO. $\uparrow P<0.05$, $\dagger \uparrow P<0.01$, at each period in $2 \mathrm{VO}$ group vs. pre operation.

\section{Figure 6.}

A, B, Histogram showing spatial working memory impairment (A) and the locomotor activity

(B) measured by Y-maze test at 28 days post-operation in the sham $(\mathrm{n}=10), 2 \mathrm{VGO}(\mathrm{n}=11)$ and $2 \mathrm{VO}(\mathrm{n}=10)$ groups.

\section{Figure 7.}

A, Representative images of Klüver-Barrera staining in the corpus callosum at 28 days post-operation in each group (upper panels). Histogram showing the grading of the white matter 
lesions in the sham $(n=4), 2$ VGO $(n=4)$ and 2VO $(n=4)$ groups. Scale bar, $50 \mu \mathrm{m}$.

B, Representative images of immunohistochemical staining for dMBP in the corpus callosum at 28 days in each group (upper panels). Oligodendrocytic cell bodies of irregular size and myelin sheaths along the axons were densely stained for dMBP. Scale bar, $20 \mu \mathrm{m}$. Histogram showing the area fraction $(\%)$ of dMBP-immunopositive fibers in the sham $(n=4), 2 \mathrm{VGO}(\mathrm{n}=4)$ and $2 \mathrm{VO}$ $(\mathrm{n}=4)$ groups.

C, Representative images of the immunohistochemical staining for glutathione S-transferase-pi (GST- $\pi$ ) in the corpus callosum at 28 days in each group (upper panels). Histogram showing the density of GST- $\pi$-immunopositive oligodendroglia in the sham $(n=4), 2 \mathrm{VGO}(\mathrm{n}=4)$ and $2 \mathrm{VO}$ $(\mathrm{n}=4)$ (lower panel) groups. Scale bar, $50 \mu \mathrm{m}$.

\section{Figure 8.}

Representative images of immunoblotting for MBP and $\beta$-actin in the optic nerve and chiasma of the sham $(n=4), 2 \mathrm{VGO}(\mathrm{n}=4)$, and $2 \mathrm{VO}(\mathrm{n}=4)$ groups at 28 days post-operation. Histogram showing the relative amount of MBP as assessed by densitometric analysis.

\section{Figure 9.}

A, B, Histogram showing the mRNA levels of TNF- $\alpha$ (A) and MCP-1 (B), as analyzed with real-time RT-PCR, in the right anterior hemisphere of the sham (normalized as 1), $2 \mathrm{VGO}$

(18.7 \pm 7.28 and $2.79 \pm 1.02$ fold, respectively), and $2 \mathrm{VO}(91.2 \pm 28.8$ and $3.68 \pm 1.14$ fold, 
respectively) groups at 1 day post-operation ( $\mathrm{n}=4$ in each group).

C, Histogram showing the protein level of TNF- $\alpha$, as analyzed with ELISA, in the left anterior hemisphere of the sham (8.38 \pm 0.63$), 2 \mathrm{VGO}(16.0 \pm 2.70)$, and $2 \mathrm{VO}(20.8 \pm 2.67)$ groups at 1 day post-operation ( $\mathrm{n}=4$ in each group). 
B

Right common carotid artery

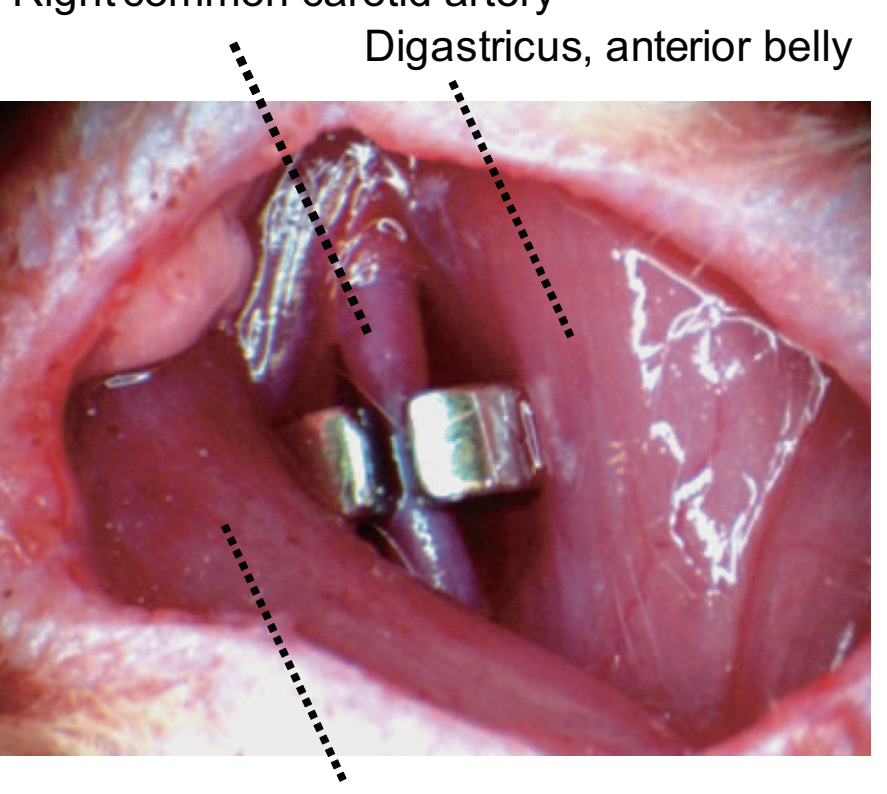

Inner diameter

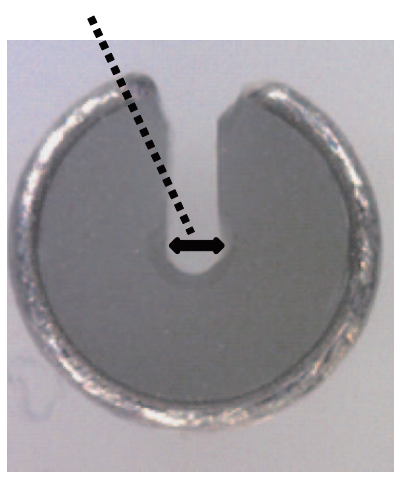

Pre

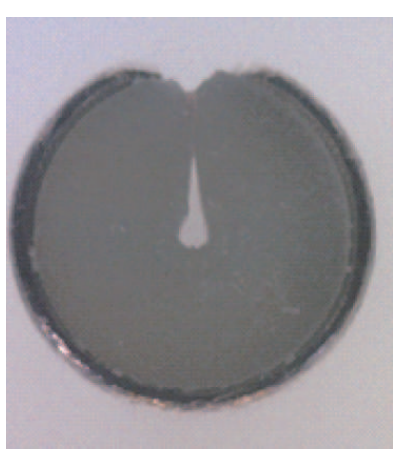

1 day

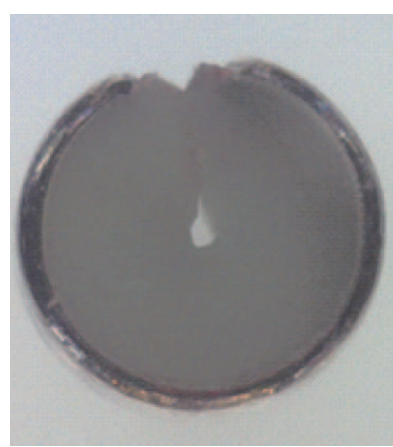

3 days
$0.50 \mathrm{~mm}$
$0.28 \mathrm{~mm}$
$0.24 \mathrm{~mm}$

Sternohyoideus

\begin{tabular}{cccc}
\hline & Pre & 1 day & 3 days \\
\hline Inner diameter $(\mathrm{mm})$ & $0.500 \pm 0.000$ & $0.282 \pm 0.00259$ & $0.241 \pm 0.00239$ \\
\hline range $(\mathrm{mm})$ & $0.500-0.500$ & $0.271-0.293$ & $0.235-0.245$ \\
\hline
\end{tabular}

Data are the mean \pm SEM.

Fig. 1 Kitamura et al. 


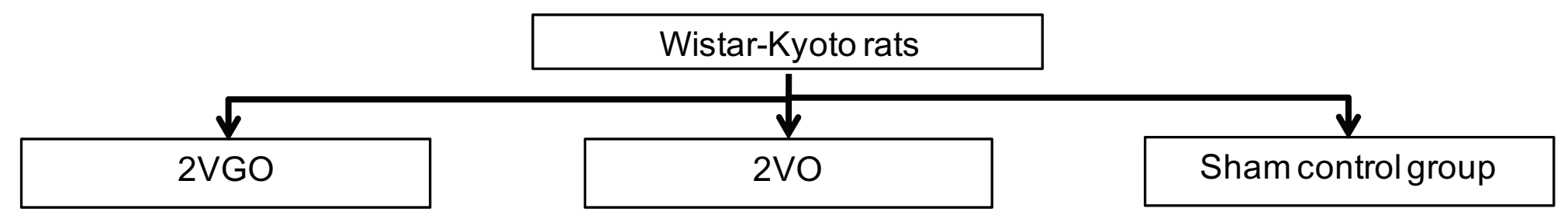

Experimental design

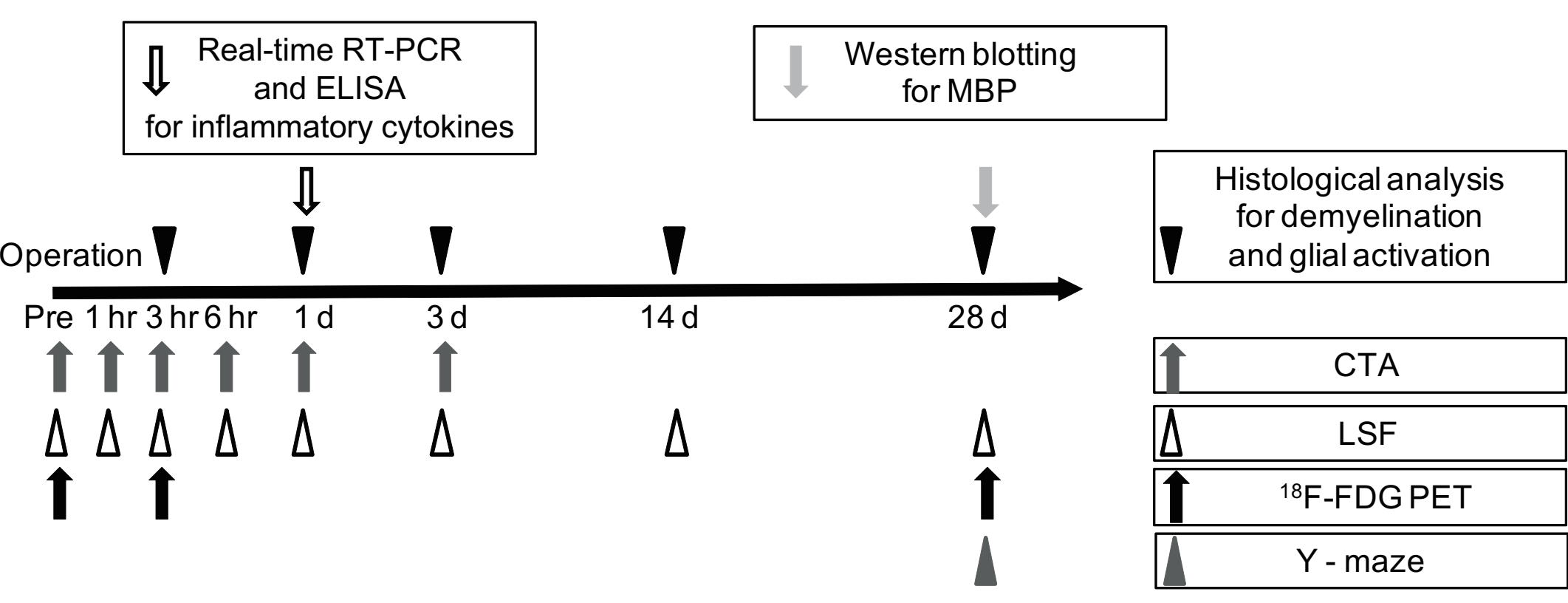

Fig. 2 Kitamura et al. 


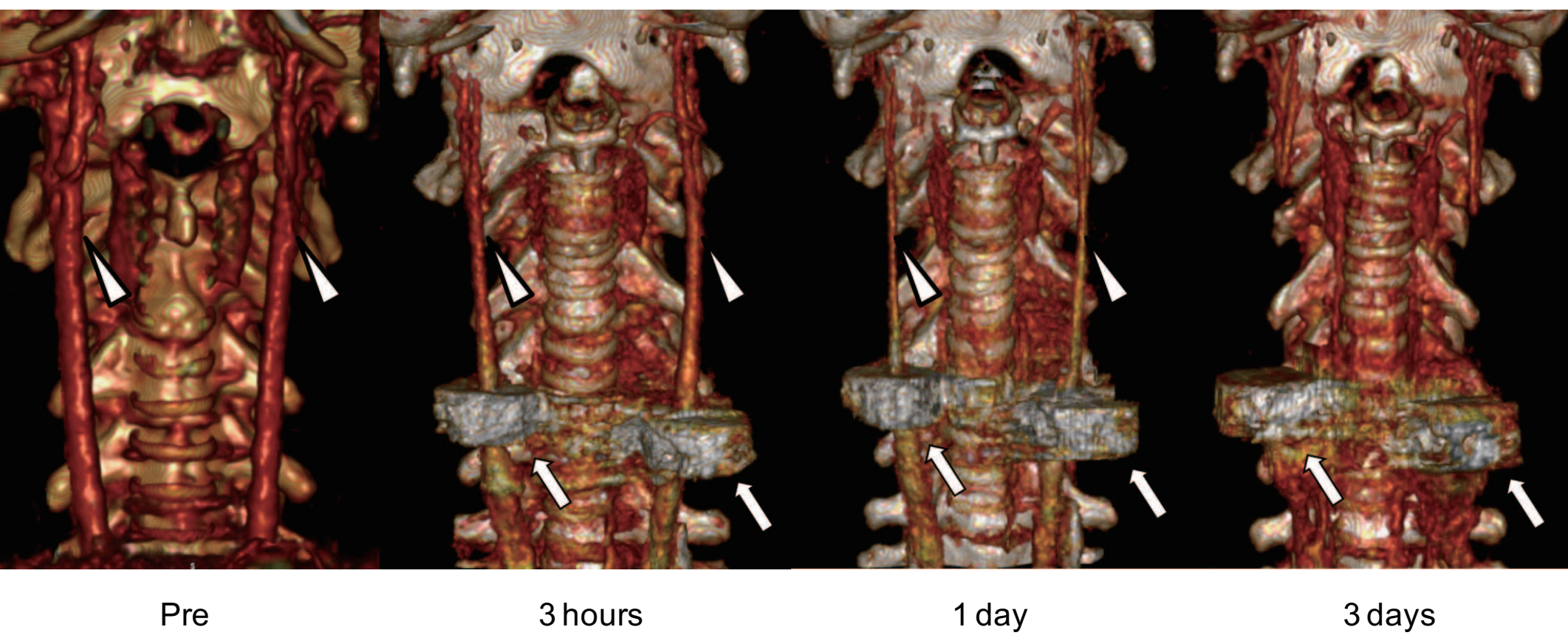

Fig. 3 Kitamura et al. 
A First 5 minutes

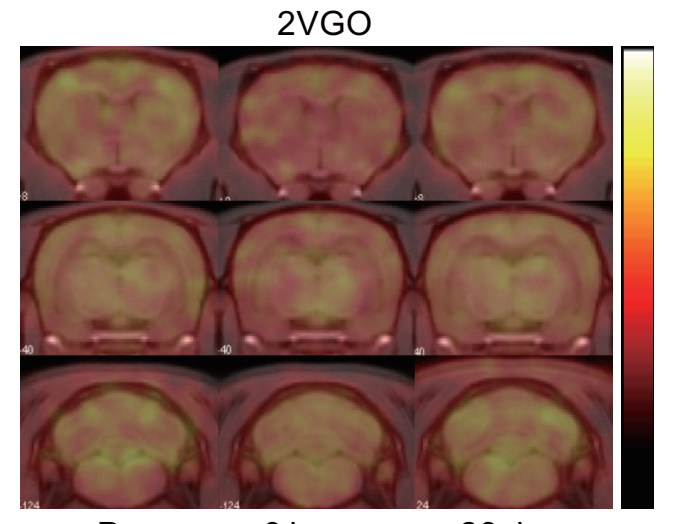

Pre
3 hours

28 days
Caudoputamen

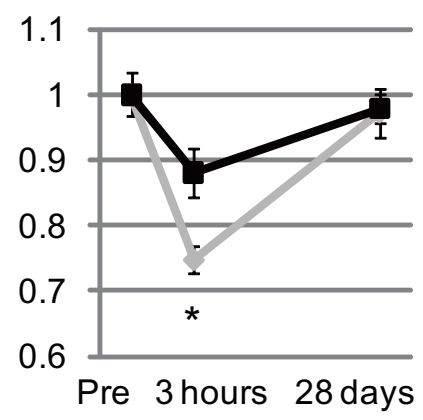

2VO

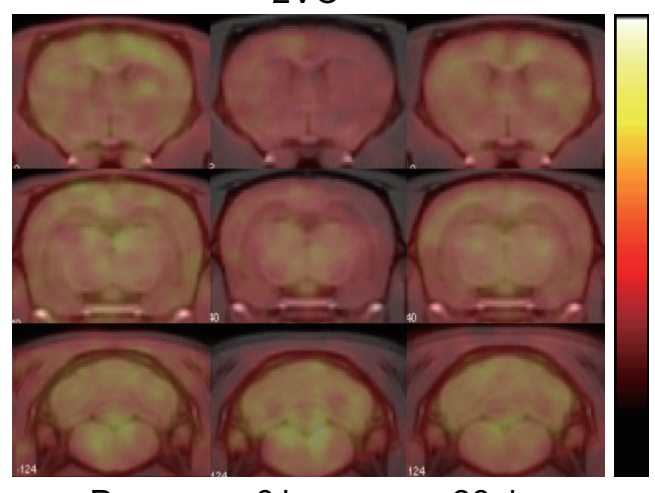

Pre
Cortex

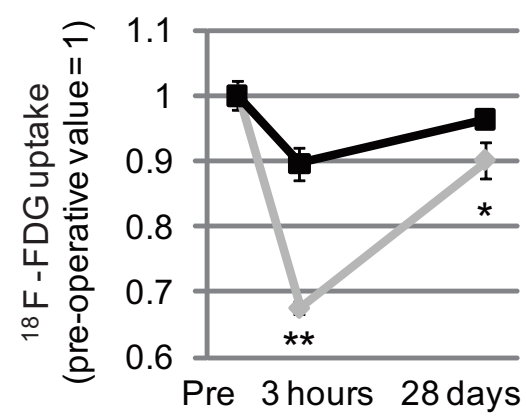

Hippocampus

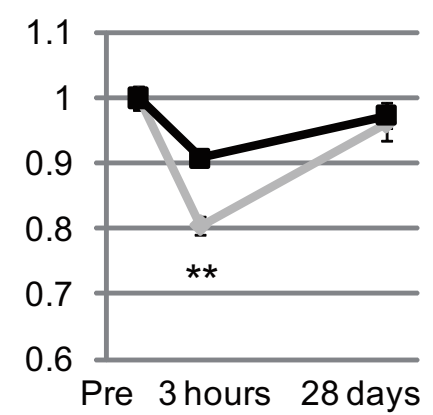

$-2 \mathrm{VGO} \leadsto 2 \mathrm{VO}$

B Between 45 and 90 minutes

2VGO

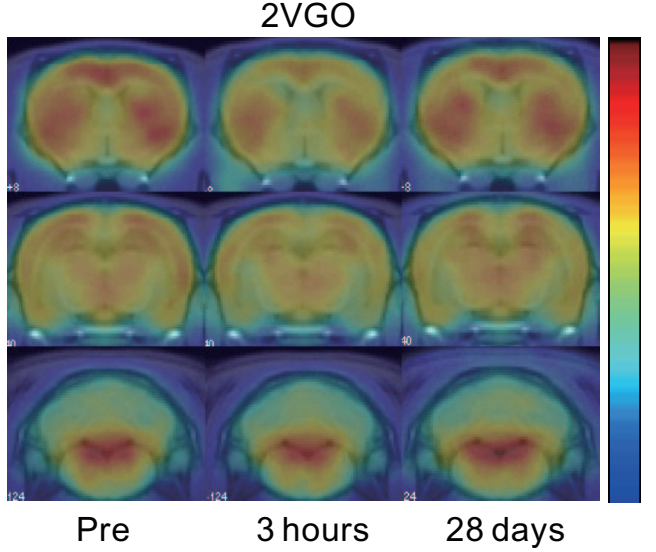

2VO

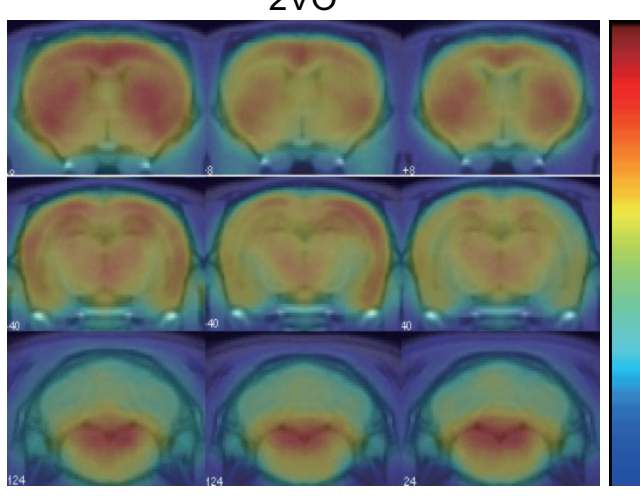

Pre

3 hours 28 days
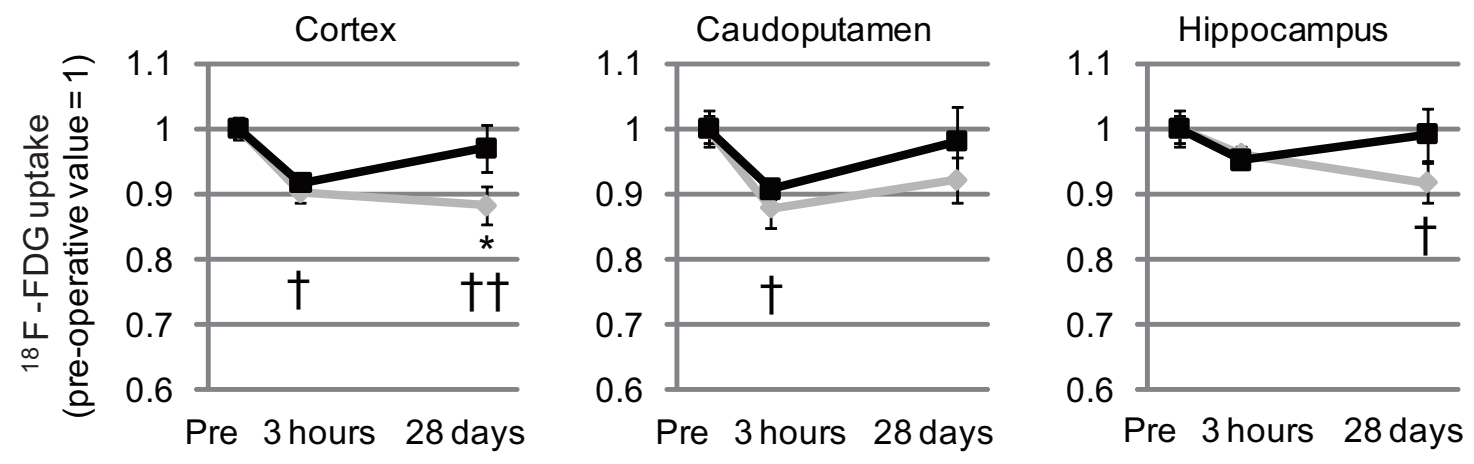

Fig. 5 Kitamura et al. 
A

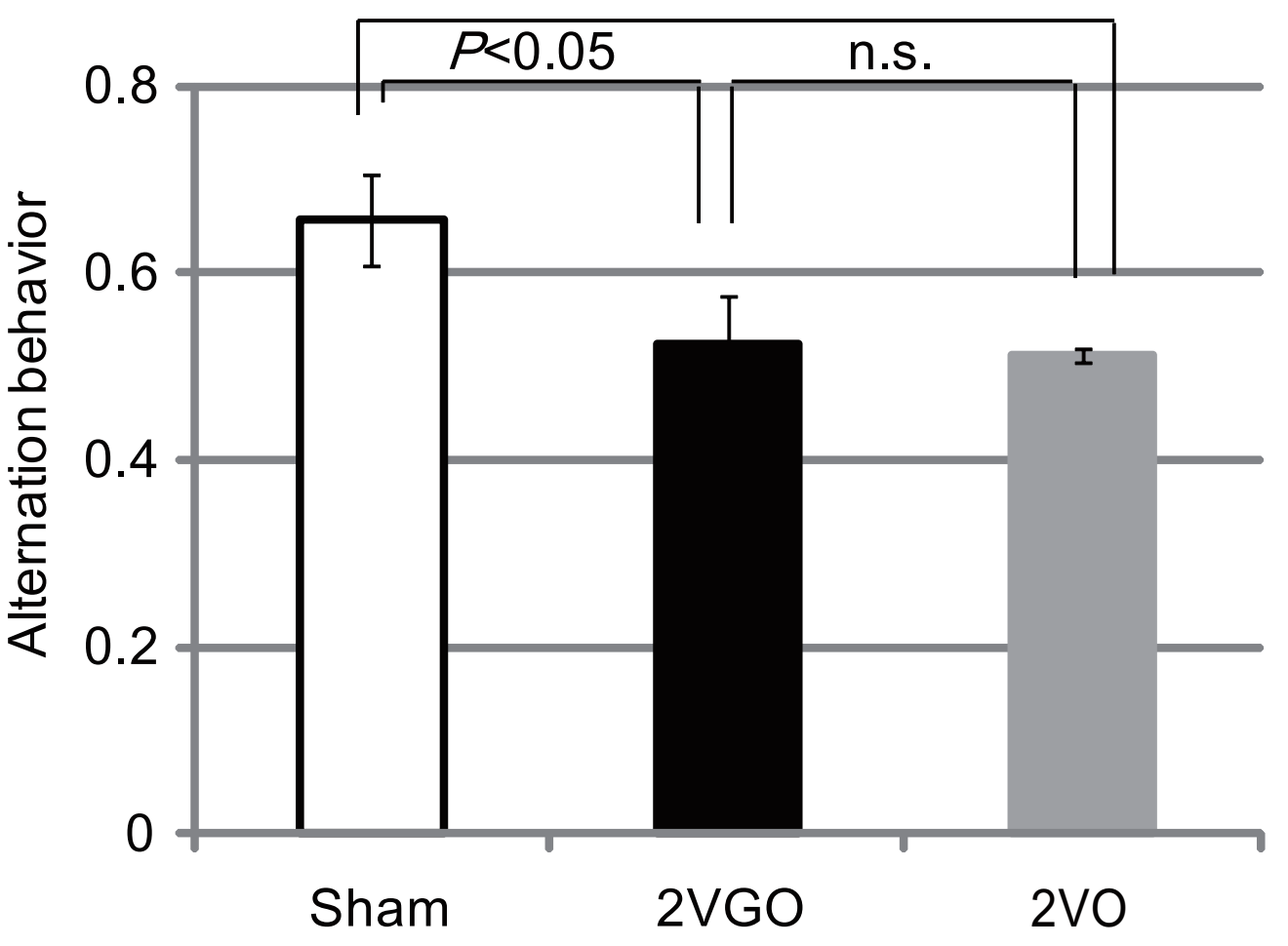

B

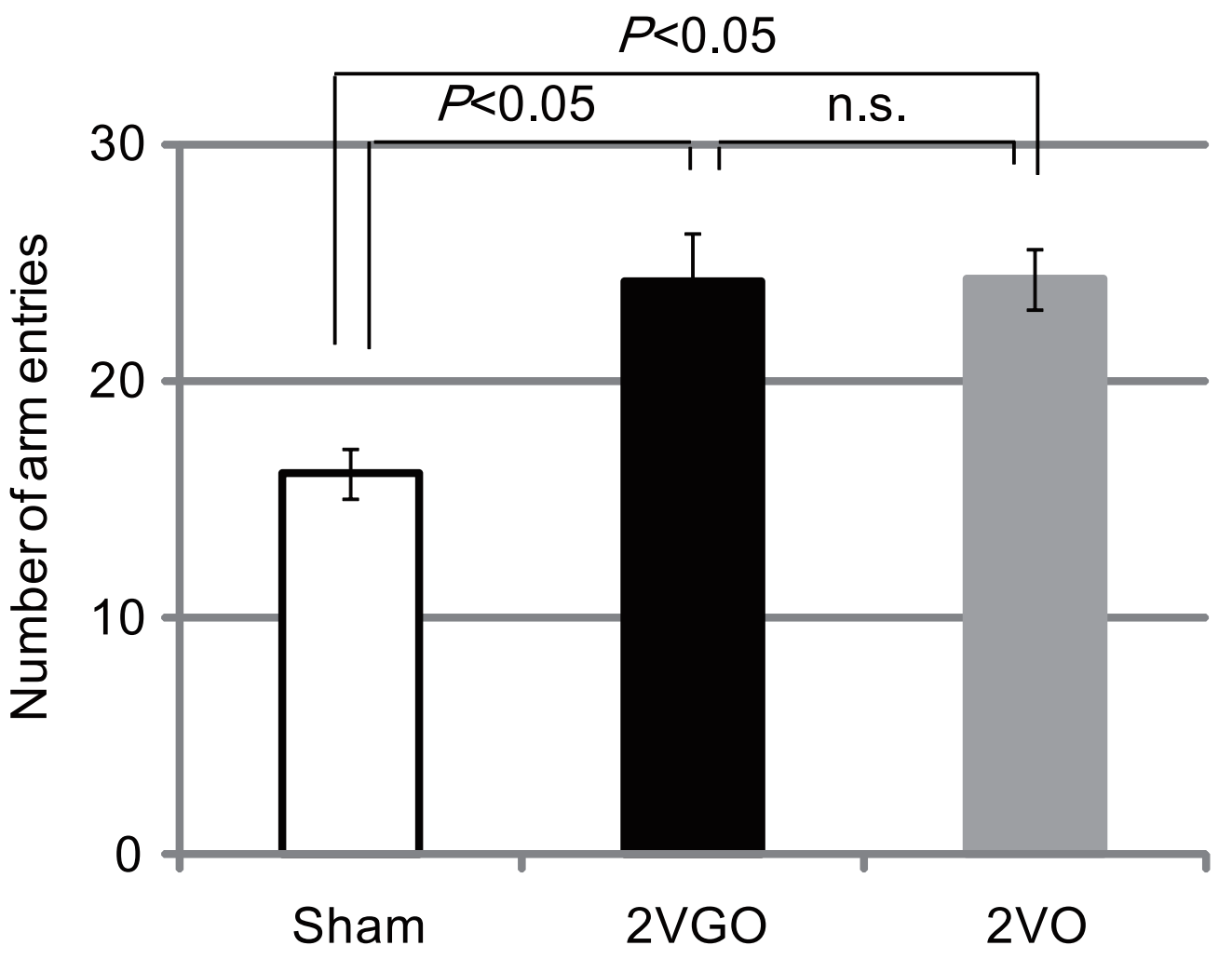

Fig. 6 Kitamura etal. 


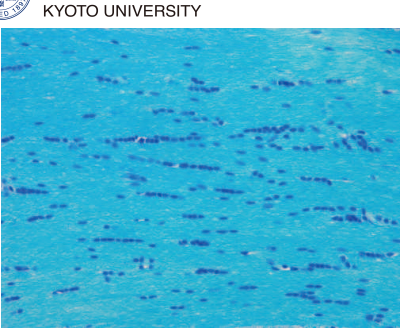

Sham

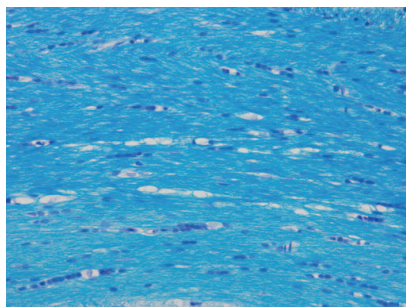

2VGO

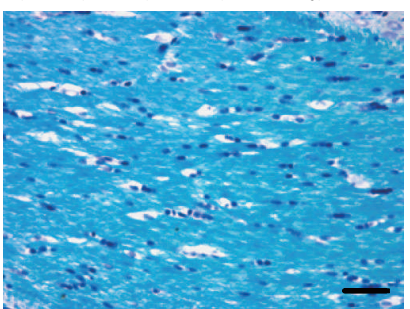

2VO

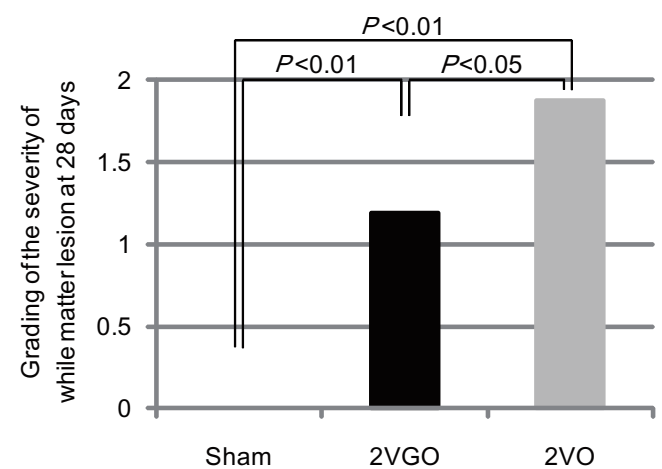

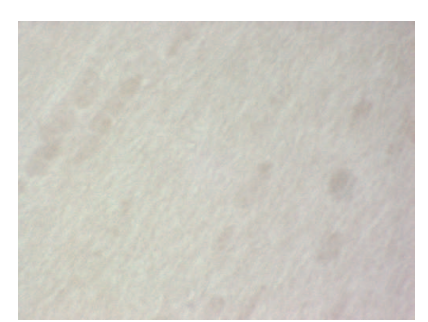

Sham

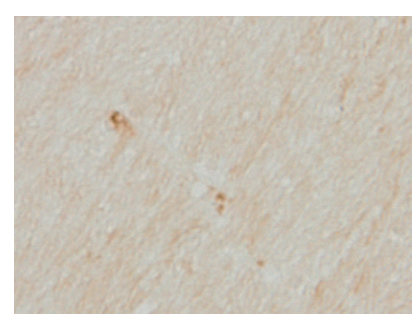

2VGO

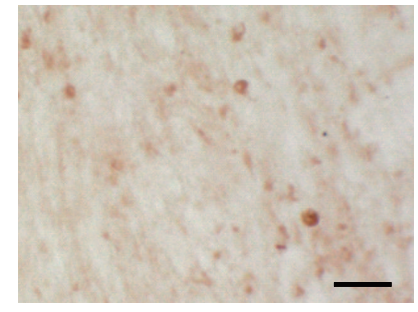

2VO

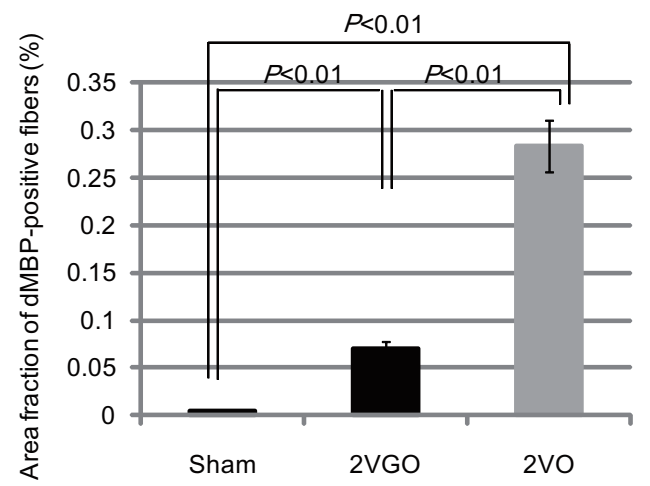

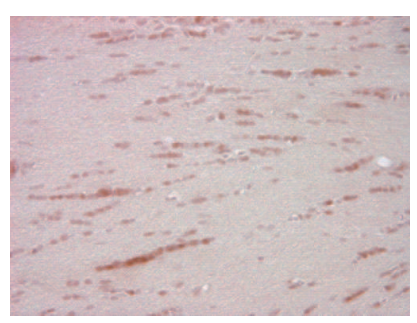

Sham

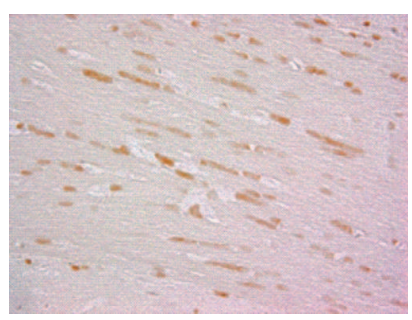

2VGO

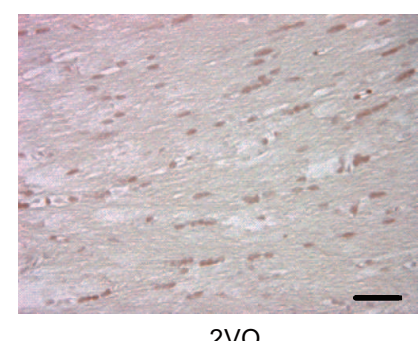

2VO

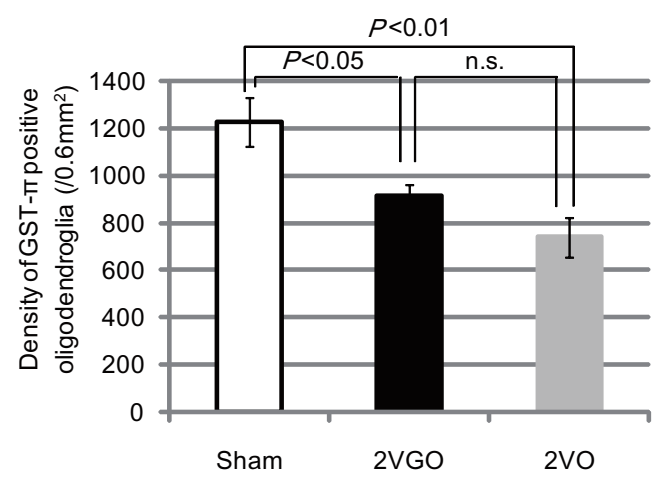

Fig. 7 Kitamura et al. 
Sham

2VGO

2VO

$\beta$-actin

$42 \mathrm{kDa}$

MBP

$21.5 \mathrm{kDa}$

$18.5 \mathrm{kDa}$

$17.0 \mathrm{kDa}$

$14.0 \mathrm{kDa}$

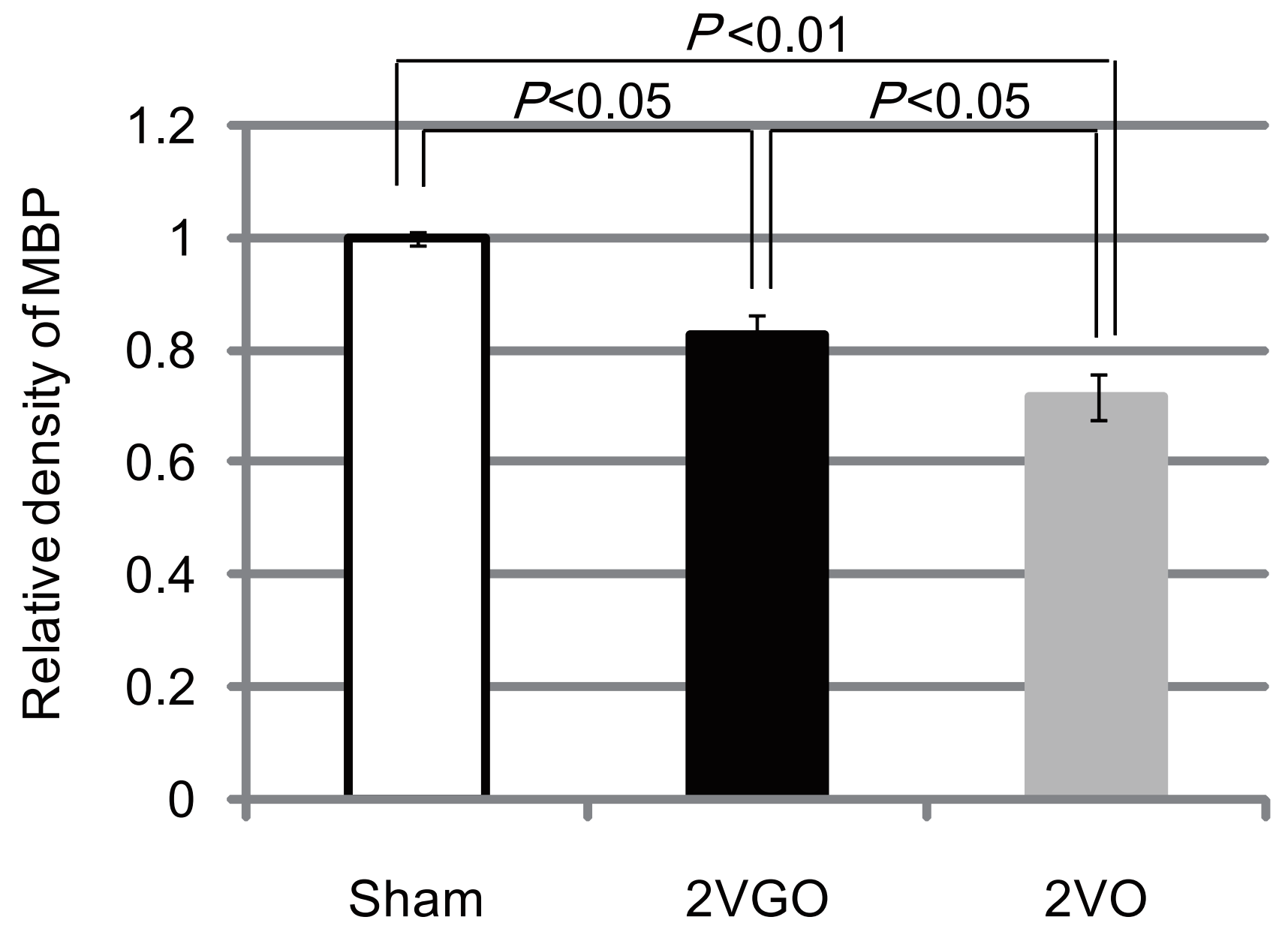

Fig. 8 Kitamura etal. 
